\title{
El desarrollo tecnológico en las revoluciones industriales
}

\author{
Technological development in industrial revolutions
}

\author{
Isidro Jesús Gonzalez-Hernandez ${ }^{a}$, Berenice Armas-Álvarez ${ }^{b}$ Melanie Coronel-Lazcano ${ }^{c}$, \\ Osmara Vergara-Martínez ${ }^{d}$ Nereida Maldonado-López ${ }^{e}$ Rafael Granillo-Macías ${ }^{f}$
}

\begin{abstract}
:
The 18th century became the witness of the first efforts of the industrial revolution; it was defined as a revolution due to its groundbreaking or disruptive nature. Besides, it was associated with the term industry since the energy source went from humans to different tools and machinery. From that moment to the present date, humanity has divided social-economic-technological development into four important epochs, known as industrial revolutions. This document aims to provide a general description of the technological evolution that has taken place in these four stages of human development through a qualitative description of a bibliographic and documentary nature to identify how manufacturing systems have evolved throughout the weather. The results reveal that the first industrial revolution has been linked to steam engines. The second revolution has been associated with electricity for assembly lines and mass production. The third revolution has been related to the computer technologies that make up automation. Finally, the fourth industrial revolution has been the massive implementation of accumulated industrial innovations and systemic transformations in the industry due to its ability to develop cyber-physical systems with advanced technologies, which translates into profound changes in logistics and manufactured products. Also, eliminating a large part of the human being in the production systems is foreseen, ensuring the complete automation of the production process.
\end{abstract}

Keywords:

Industrial revolutions, Industry 4.0, manufacturing systems

Resumen:

El siglo XVIII se convirtió en el testigo de los primeros esfuerzos de la revolución industrial, se definió como una revolución debido a su naturaleza rompedora o disruptiva. Además, se asoció con el término industria, ya que la fuente de energía pasó de los humanos a las diferentes herramientas y maquinarias. Desde ese momento hasta la fecha actual, la humanidad ha dividido en 4 épocas importantes el desarrollo social-económico-tecnológico, conocidas como revoluciones industriales. El objetivo de este documento es brindar una descripción general de la evolución tecnológica que se ha llevado a cabo en estas cuatro et apas del desarrollo humano, mediante una descripción cualitativa de carácter bibliográfico y documental para identificar cómo han evolucionado los sistemas de manufactura a lo largo del tiempo. Los resultados revelan que la primera revolución industrial se ha vinculado a las máquinas de vapor. La segunda revolución se ha asociado a la electricidad para líneas de montaje y producción en masa. La tercera revolución se ha relacionado con las tecnologías informáticas que forman la automatización. Por último, la cuarta revolución industrial ha sido la implementación masiva de innovaciones industriales acumuladas y transformaciones sistémicas en la industria, debido a su capacidad de desarrollar sistemas ciberfísicos con la aplicación de tecnologías avanzadas, lo que se traduce en profundos cambios en la logística y los productos manufacturados. Además, se prevé la eliminación de gran parte del ser humano en los sistemas de producción, asegurando la automatización absoluta del proceso de producción.

\section{Palabras Clave:}

Revoluciones industriales, Industria 4.0, sistemas de manufactura

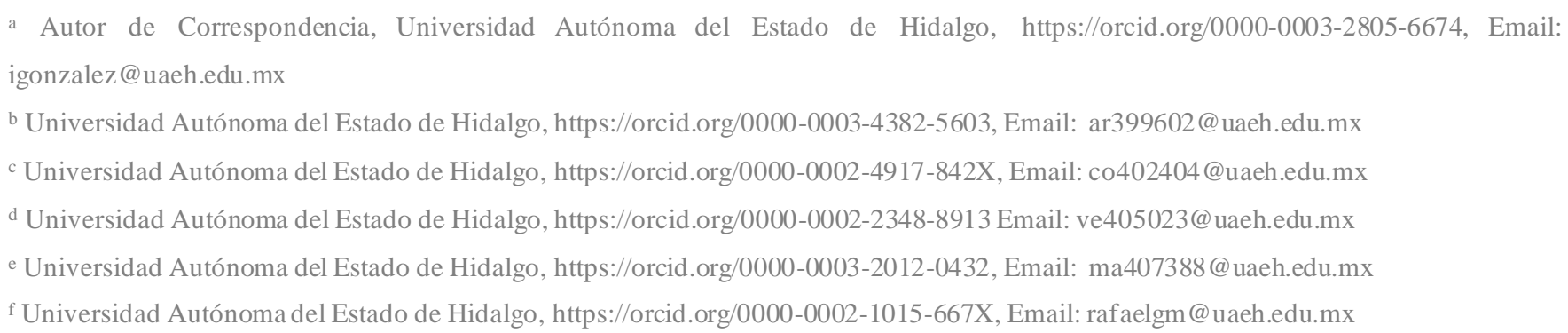




\section{Introducción}

El término revolución proviene del vocablo latino revolutio, "una vuelta", "inquietud", "revuelo" o "alboroto", al mismo tiempo usado también como "cambio", "renovación" o "vanguardia", se refiere en sí a un cambio social organizado, masivo, intenso, repentino y generalmente no exento de conflictos violentos para la alteración de un sistema político, gubernamental o inclusive económico (SIGNIFICADOS,2019).

La Revolución Industrial se conoce más habitualmente en la actualidad como el período histórico de cambio cíclico, irreversible y rotativo, caracterizado por transformaciones radicales y repentinas en diversos ámbitos de la sociedad, implica un momento singular de ruptura y establecimiento de un a frontera temporal clara y tortuosa, entre el pasado y el desarrollo del futuro. Este importante acontecimiento de industrialización que marcó un antes y un después en la historia de la humanidad comenzó en Inglaterra a finales del siglo XVIII y continúa hasta nuestros días (Selva, 2016). Durante este período, se desarrolló la mecanización de la agricultura, la industria textil, surgió la maquinaria de transporte y comunicaciones, como barcos, trenes, telégrafo y el teléfono, se mejoró la productividad de las industrias, aumentó notablemente la economía y dio apertura a la globalización y conexión entre países. Estos cambios tuvieron consecuencias positivas no solo en el ámbito social y cultural, sino también en el económico e industrial. Como parte de esta revolución, las condiciones laborales globales también tuvieron un cambio radical (González, 2021).

Se le conoce como Primera Revolución Industrial a la creación de innovaciones tecnológicas y científicas que supusieron ruptura con las estructuras socioeconómicas existentes hasta ese momento; después de que años más tarde se produjera una nueva revolución industrial que la derrocaría, conocida como Segunda Revolución Industrial. En los siglos XX y XXI se produjeron la Tercera y la Cuarta, respectivamente (Selva, 2016).

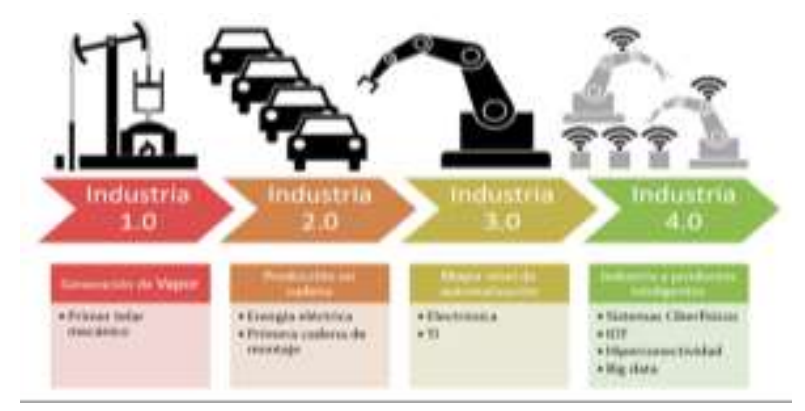

Figura 1. Evolución de la industria 1.0 a 4.0

Fuente: Avansis (2021)
Antes de que estallara la Primera Revolución Industrial, todos los productos eran manufacturados artesanalmente en pequeños talleres o en las casas; los fabricantes de estos productos eran principalmente personas con conocimientos previos sobre el oficio que se dedicaban únicamente a la producción de estos artículos. La demanda era controlada y mensurable ya que no se contaba con la suficiente capacidad productiva, además de que la comercialización era solamente en mercados locales. Sin embargo, la creación de empresas y el aumento de demanda de productos orillaron a que el conocimiento buscará cómo cubrir esas necesidades por lo que comenzó la creación de máquinas y herramientas que a su vez mejorarían los sistemas de producción y permitirían avanzar rápidamente en la generación de productos elaborados a pequeña escala (BARDAHL, 2020). Pero no sólo contribuyó a ese avance, sino que además fue la causante directa de muchos otros avances que hoy en día se dan por sentado, como por ejemplo el transporte, la electricidad, los electrodomésticos, que hasta el día de hoy son esenciales para nuestra calidad de vida.

Se puede decir que la revolución industrial es el resultado de un extenso y duradero proceso de creación e innovación humana que muestra claramente la importancia de la intervención de la tecnología y la ciencia y las mejoras que se derivaron, derivan y derivarán de ellas (Bembibre, 2014).

\section{Método}

La metodología de este trabajo incluye una investigación documental basada inicialmente en una revisión de la literatura sobre dos aspectos: 1) la evolución de las revoluciones industriales para identificar cuáles fueron las principales tecnologías que se desarrollaron para mejorar los sistemas de producción. 2) Identificar cuáles son las tendencias de los sistemas de manufactura y producción en la Industria 4.0.

\section{Primera revolución industrial}

Qué debemos entender por Revolución Industrial, de acuerdo al profesor británico David S. Landes, el término revolución industrial suele referirse al complejo de innovaciones tecnológicas que, al sustituir la habilidad humana por la maquinaria y la fuerza humana y animal por energía mecánica, provoca el paso desde la producción artesana a la fabril, dando así lugar al nacimiento de la economía moderna. De acuerdo con esa interpretación, una sociedad industrial es aquella que sabe aprovecharel desarrollo tecnológico para sustituir la energía proporcionada por músculos humanos o 
animales, o también turbinas, por la proporcionada por máquinas. (Chaves, 2014)

La etapa de la primera revolución va desde 1780 a 1840 que consiste en una transformación profunda, que engloba los sistemas de trabajo y la estructura de la sociedad. Si bien se sabe que esta revolución se expresó primero en Inglaterra y en menor medida en Francia y Holanda, se aplicó el método tecnológico a diferentes problemas desde económicos, sociales, políticos hasta los industriales (Coluccia, 2012).

Chaves (2014) menciona que el resto de las naciones desarrolladas se industrializaron a lo largo del siglo XIX siguiendo el ejemplo preexistente de Gran Bretaña, en donde los inventos y el progreso industrial estuvo fuertemente mediatizado por el influjo inglés. En América del Norte y resto de Europa iniciaron su andar en la revolución industrial con la tecnología facilitada por Inglaterra, también con trabajadores ingleses que fueron demandados por esos países para que los instruyeran en la utilización de los nuevos motores y maquinarias, e incluso, en no pocas ocasiones, recurrieron al capital inglés para hacer frente a las operaciones financieras que facilitarán las inversiones inherentes a todo el proceso de industrialización.

Entre los hechos más importantes destacaron las políticas mercantiles adoptadas por las naciones líderes en el comercio, las cuales condujeron a una serie de conquistas coloniales mediante la acción militar. En el siglo XVIII se intensificó la importación de oro y plata, y se obtuvieron ganancias de la producción de azúcar y el trabajo de esclavos estadounidenses. Los beneficios de la producción y el comercio colonial fueron muy importantes y debido a esto entraron en conflicto empresas inglesas y francesas. Posteriormente América del Norte logró un progreso económico considerable con su sistema agrícola-comercial-manufacturero (Coluccia, 2012).

Estados Unidos, el 4 de julio de 1776, el Congreso de las Colonias norteamericanas votó a favor de la declaración de independencia de Estados Unidos, dicha petición desató una guerra con una duración aproximadamente de seis años, logrando así la independencia.

La independencia de las colonias norteamericanas abrió el camino a otros movimientos de liberación, algunos de los cuales tuvieron un resultado positivo: por ejemplo, Argentina 1816, Colombia 1819, México, 1821. Otro de los hechos más significativos fue el movimiento revolucionario de 1789 que derrocaron a la monarquía, desmantelaron muchos de sus privilegios y abolieron algunos monopolios y ciertos privilegios de las empresas comerciales. Las clases medias altas fueron las que se vieron principalmente satisfechas al acceder a los más altos cargos parlamentarios, el poder judicial, el ejército y la administración. Por otro lado, los campesinos, los trabajadores asalariados, los pequeños artesanos y los pequeños comerciantes estuvieron decepcionados al observar la victoria de las clases medias y altas.

El final del siglo XVIII (especialmente los últimos 30 años) marcó el comienzo de la Primera Revolución Industrial, que provocó profundos cambios sociales, políticos y económicos lo cuales llevaron un proceso que ya había comenzado desde tiempo atrás consolidándose en los últimos años del siglo XVII (Coluccia, 2012).

La primera revolución industrial se caracteriza como un proceso largo, complejo y sistemático que provocó el cambio de la manufactura en pequeña escala, a la industria artesanal y a empresas con trabajadores asalariados, que utilizaban mano de obra en la producción, incluso fábricas dirigidas por empresarios capitalistas que también asumen los riesgos de la inversión a gran escala, ya que hicieron uso significativo de máquinas impulsadas por energía hidráulica o vapor. Otro hecho fue el movimiento de la población de las comunidades rurales hacia las urbanas. Además de la ampliación y despersonalización de una unidad típica de producción, pasó a fundarse más en la empresa privada o pública y menos en la familia o la tribu. De igual manera, el movimiento de la mano de obra de las actividades relacionadas con la producción de bienes primarios a la producción de bienes manufacturados y servicios se manifestó (Coluccia, 2012).

A su vez, los avances tecnológicos y empresariales continuaron una dirección convergente. De tal forma que un cambio generaba otro cambio. En este sentido cabe mencionar otra de las características de la primera revolución industrial, la cual se basa en el principio de que, una vez iniciado el proceso, éste se prolonga indefinidamente. El "despegue" conduce al "desarrollo que se sostiene a sí mismo". Ya que, un producto nuevo crea la demanda de otros. Una invención da origen a la siguiente y la misma invención se convierte en un hábito. Esa dinámica se aprecia con claridad en las mejoras tecnológicas y su dependencia de los avances en otras actividades afines. (Coluccia, 2012).

Con respecto a las innovaciones científicas en la primera revolución industrial, el desarrollo de la ciencia y la tecnología fue en el periodo de la ilu stración y del análisis racional de los problemas. Así como, en la aplicación de principios científicos a los procesos de producción y de los avances tecnológicos en la agricultura durante la década de 1700 (Coluccia, 2012).

Por otra parte, en la manufactura donde se tuvieron nuevas formas de organización tecnológica y económica de la producción industrial, la organización técnica y económica de la producción industrial continuaría vigente hasta fines de la tercera década del siglo XIX, donde se estructuró de la siguiente manera: 
- Artesanos que habitualmente trabajaban con un maestro artesano empresario o con un comerciante que financiaba y organizaba la producción con el objetivo de vender los productos.

- Industria artesanal realizada en zonas rurales, distribuida en aldeas y ocupada por familias que también se dedicaban a la agricultura. Este tipo de fabricación también se realizaba normalmente bajo la supervisión de un comerciante que organizaba y financiaba la producción y la venta (Coluccia, 2012).

- Instalaciones de fabricación que eran propiedad de un comerciante empresario que empleaba a trabajadores asalariados, que realizan principalmente trabajos manuales (Coluccia, 2012).

El mayor impacto en la manufactura y tecnología fueron en el algodón, lana, industria minera y las ferreteras. Dentro del sector de algodón tenemos:

- El transbordador volador de John Kay (1733, adoptado en 1760) fue una mejora del telar manual (para tejer) introducido en la industria de las cotizaciones. No condujo a la construcción de fábricas.

- La hiladora Jenny de James Hargreaves (1764), que mejoró la productividad del hilado; como en el caso anterior, las máquinas no condujeron a la construcción de fábricas ya que fueron utilizadas por pequeñas industrias artesanales rurales.

- La aplicación de la fuerza del agua por Richard Arkwright para impulsar máquinas (ruedas giratorias) para girar. Esta invención tuvo lugar en 1767, y la aplicación (1779) tuvo una importancia histórica, ya que fue acompañada por el desarrollo de la gran fábrica (300 empleados para la hilandería mecanizada) (Coluccia, 2012).

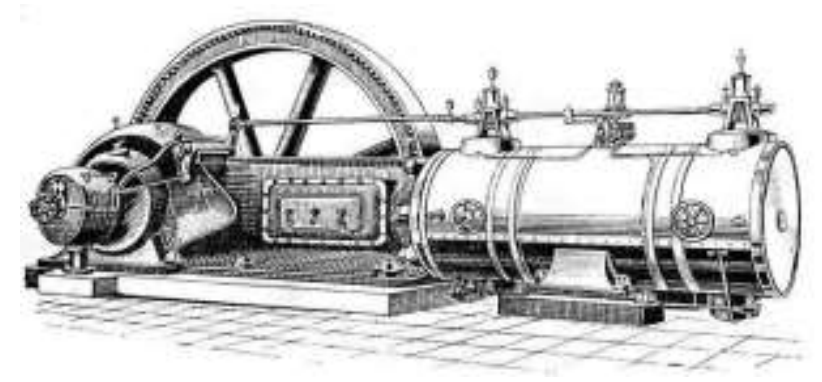

Figura 2. Maquina a vapor

Fuente: Riedel (2016)

La fecha de 1779 puede considerarse decisiva en la historia de la industria algodonera inglesa porque a partir de esta fecha se inició la marcha triunfal del hilado mecanizado. Esta fue la condición primaria e indispensable para permitir que la nueva industria textil comenzará su maravillosa expansión. A partir de 1779, se puede describir como surgimiento de la nueva organización técnica y económica de la producción industrial, es decir la nueva forma de fabricación. En los 20 años siguientes, el hilado manual (realizado con fines comerciales) desapareció casi por completo y fue reemplazado por el hilado mecánico, que era cuarenta veces más productivo; en el tejido, el telar manual fue sustituido por el marco mecánico inventado por Edmund Cartwright en 1785. Sin embargo, su verdadera aplicación industrial no tuvo lugar hasta 1806, en Manchester, cuando se inició la primera actividad de tejido mecanizado. En este sector, la innovación se produjo más lentamente, de modo que incluso en 1830 los telares mecánicos utilizados en la industria del algodón representaban solo el 20 por ciento del número total en toda Inglaterra.

En el sector de lana en 1800 se introdujo máquinas para trillar, cardar y peinar lana, en 1830 se introdujeron procesos mecanizados y de igual manera el uso de agua para producir energía y la construcción de máquinas de hierro y acero en lugar de la madera. James Watt patentó la primera máquina de vapor en 1764 posteriormente en 1781, Watt patentó un nuevo invento que transformó la máquina de vapor en un motor que se podía utilizar en todo tipo de aplicaciones (Coluccia, 2012).

Las innovaciones científicas y tecnológicas descritas anteriormente se concentraron entre las fases finales del siglo XVIII y las tres primeras décadas del siglo XIX, especialmente debido a una serie de factores que se unieron para crear un sistema. La investigación y las nuevas innovaciones tecnológicas encontraron una pronta aplicación porque en ese período las condiciones eran ideales para la aplicación de ciertas ideas. Leonardo da Vinci (1452-1519) llevó a cabo una investigación que lo llevó a descubrimientos geniales: máquinas voladoras, primitivos vehículos blindados, bicicletas con cadenas y ruedas dentadas y muchos otros dispositivos mecánicos. Pero estas ideas, con sus asombrosas implicaciones, nunca se aplicaron porque en ese período las condiciones no eran las adecuadas para su aplicación (Coluccia, 2012).

Los principales catalizadores de la aplicación de la ciencia a la actividad productiva en Inglaterra fueron los siguientes: 1) el aumento demográfico significativo durante el siglo XVIII, consiguiendo así diversas fuentes de empleo, mejoras en los sistemas y medios de transporte para que así fuesen más rápidos y eficientes; 2) la explotación de colonias teniendo un importante desarrollo en el comercio internacional de materias primas y productos manufacturados: 3) la obtención de 
capital extranjero para la financiación de minas, industrias y comercio; 4) el aumento en la demanda nacional e internacional de cereales y productos de lana y algodón. Estos catalizadores estimularon la investigación y tecnología innovadora para la mejora en la calidad de los productos reduciendo significativamente los costos (Coluccia, 2012).

\section{Segunda revolución}

La Segunda Revolución Industrial por lo general suele reconocerse como un proceso que tuvo lugar entre los años 1870 y 1914. Aunque algunos de sus eventos característicos se remontan a la década de 1850. Está claro que el rápido desarrollo de invenciones pioneras se produjo después de 1825 y que tuvieron gran impacto en años posteriores (Mokyr, 1999).

Esta importante transformación fue la consecuencia de la histórica invención de la máquina de vapor durante el desarrollo de la primera revolución industrial. La energía utilizada por esta máquina, promovió intensamente la productividad, simultáneamente el progreso de la producción en masa y la mecanización de la industria, pero las deficiencias de esta energía fueron crecientes por lo que un mayor desarrollo industrial requería una fuente de energía más conveniente; esto se convirtió en el avance para la búsqueda de nuevas alternativas ante esta necesidad, promoviendo así el comienzo de la Segunda Revolución Industrial (Zhang y Yang, 1999). Otro factor importante que dio apertura al desarrollo de esta revolución durante el año 1830, fue que los nuevos medios de transporte, como el ferrocarril y los barcos de vapor, se habían extendido enormemente. Lo que favoreció la llegada de todo tipo de productos a distintos mercados. Además de que, otros países comenzaron sus propios procesos de industrialización e iniciaron la explotación de recursos mineros necesarios para las industrias, como el carbón y el hierro, y la fabricación de maquinaria. Así comenzó una nueva etapa de la industrialización, caracterizada por el aprovechamiento de recursos y nuevas fuentes de energía (KAPELUSZ, 2020).

En la segunda revolución industrial se dio pauta a que existiera mayor interrelación entre la ciencia y la tecnología, además de ser uno de los cambios más significativos a lo largo de toda la historia. De esta manera la innovación tecnológica ganó modernidad y sentó las bases tecnológicas del siglo $\mathrm{XX}$, manteniéndose al margen las bases impuestas por la primera revolución industrial, que tenían carencia sobre el conocimiento científico.

La ciencia y la tecnología de este período se caracterizaron por la aplicación de su entendimiento para la creación de máquinas y equipos con mayor complejidad, pero la relación más estrecha entre ambos, requirió mayores calificaciones para su implementación y suscitó la participación de más científicos, desplazando las formas menos formales de experiencia e información empleadas en la revolución predecesora.

Todos estos descubrimientos e invenciones finalmente formaron un nuevo sistema técnico. El resultado del nuevo sistema era ampliar los recursos naturales disponibles, desarrollar otras innovaciones tecnológicas complementarias y ahorrar mano de obra, lo que aumentaría enormemente la productividad, las ganancias, los salarios, precios bajos para el consumidor y lanzamiento de nuevos productos al mercado. El éxito de las invenciones de la segunda revolución industrial fue más amplio que el de la primera, otorgando una mayor variedad de productos y actividades. El nivel de vida y el poder adquisitivo del dinero aumentaron rápidamente debido a que las nuevas tecnologías se están extendiendo a la vida cotidiana de la clase media y la clase trabajadora. En el último tercio del tiempo, la continuidad y aceleración del progreso tecnológico se atribuyó cada vez más a la acumulación continua de conocimientos útiles otorgados por la ciencia (Mokyr, 1999).

A principios de 1870 surge la nueva industria del metal para satisfacer la creación masiva de trenes y ferrocarriles necesarios para el transporte. Crece la industria química, de creación y distribución de bienes petroleros. La industria eléctrica también se encontraba en desarrollo y a inicios del siglo XX, el surgimiento de la industria automotriz complementó la segunda revolución Industrial (González, 2021).

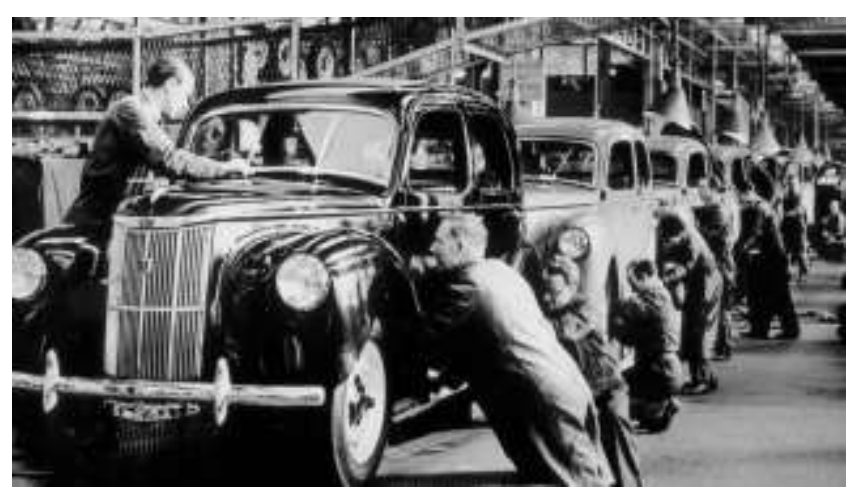

Figura 3. La teoría del Taylorismo

Fuente: QAlovers (2017)

En ese momento, el progreso de la tecnología y la ciencia comenzó a mostrar una transición más compleja. De esta forma se comenzaron a obtener diferentes recursos naturales que no estaban disponibles o no eran muy útiles antes de ese momento. Entre ellos, particularmente metales como acero, zinc, aluminio, níquel o cobre. 
Además, el desarrollo de la industria ferroviaria originó que se utilizarán productos químicos y comenzaron a aparecer nuevas formas de energía, como la electricidad o el uso del petróleo, precedidas del avance tecnológico y científico. En este sentido, se puede hablar de la máquina de turbinas o de la industria del gas (Selva, 2016).

La segunda revolución industrial originó un cambió radicalmente en el orden económico mundial que se suscitó en esa época, donde Estados Unidos y Alemania se convirtieron en los principales productores industriales del mundo (González, 2021).

Las principales características de la segunda revolución industrial, fueron la consolidación de un nuevo modelo de capitalismo, que tendía a ser monopolista. Por otro lado, la necesidad de buscar nuevos mercados llevó a la aceleración de proyectos imperialistas. En esta nueva situación, las potencias industriales compitieron por conquistar nuevos territorios y expandir mercados, lo que generó una franca tensión e incluso el estallido de la Primera Guerra Mundial (Selva, 2016).

Paralelamente la ciencia durante esta revolución industrial pasó a ser muy relevante e impactante, volviéndose un pilar por delante de las innovaciones tecnológicas, las teorías y experimentos de la ciencia tomaron mucha importancia debido a que debía tenerse mayor conocimiento para desarrollar sistemas más eficientes o para la obtención de energías o materiales nuevos; la electricidad, el gas natural y los derivados del petróleo comenzaron a utilizarse como nuevas fuentes de energía, se empezaron a utilizar recursos y aleaciones como el acero, el carbón o el aluminio. Además, la investigación científica se comenzó a aplicar en la industria (Zhang y Yang, 1999).

La Segunda Revolución expandió la industrialización a las industrias pesadas, como el hierro y el acero, y la construcción de maquinaria, etc. Además, surgieron y se desarrollaron algunas industrias nuevas, como la petrolera, química, eléctrica, automotriz, etc. Se produjeron avances importantes en los campos de la automoción y las comunicaciones. Igualmente se implementaron máquinas automatizadas utilizadas en la industria a gran escala y la producción en masa utilizada como sistema de trabajo (Zhang y Yang, 1999).

El uso de la electricidad fue facilitado por la creación de tres tipos de maquinaria: la primera eran las turbinas, las cuales eran capaces de generar la energía eléctrica. La segunda eran los acumuladores eléctricos, que permitían transportar la electricidad. La tercera eran los motores, capaces de convertirla en energía productiva para las máquinas (Zhang y Yang, 1999).

El aumento de la producción en los países industrializados impulsó la búsqueda de más mercados. Se consolidó, entonces, un mercado mundial en el que los países industrializados y los países no industrializados asumieron funciones diferentes. Mientras que los países industrializados se ocupaban de vender productos elaborados, los no industrializados se encargaban de ofrecerles materias primas, como metales y algodón, y alimentos, como carnes, café, frutos tropicales, entre otros. Además, los países industrializados enviaban capitales hacia los países no industrializados para invertirlos en actividades que aseguraran la producción de materias primas y alimentos. Por ejemplo, inversiones en ferrocarriles, bancos y mejoras en los puertos.

También en este período se produjeron migraciones desde Europa hacia América, especialmente a Estados Unidos, la Argentina y Brasil, donde los recién llegados se incorporaban como trabajadores (Zhang y Yang, 1999).

La competencia entre potencias también se manifestó en la construcción de dos canales, el de Suez (1869) y el de Panamá (1914), que facilitaban la comunicación entre los océanos y, por lo tanto, agilizaban el comercio (KAPELUSZ, 2020). Así mismo, el desarrollo de las telecomunicaciones y transportes tuvo gran auge, esto se inició con la aplicación de la máquina de vapor al transporte marítimo (barco de vapor, 1807) y terrestre (ferrocarril, 1825). Durante la segunda mitad del siglo XIX recibieron un impulso fundamental que los extendió por todo el planeta.

La sustitución del hierro por el acero mejoró la resistencia de los raíles y, por tanto, la velocidad, la seguridad y la capacidad de carga de los vagones. Los costos se abarataron y el ferrocarril se convirtió en el sistema de transporte de personas y mercancías más utilizado (Monllor, 2012).

Al igual los descubrimientos en telecomunicaciones más destacados durante el proceso de la segunda revolución industrial fueron: el telégrafo, que fue construido en 1837 por Samuel Morse, denominado "código morse". Este artefacto permitía una comunicación al instante mediante un idioma de puntos y líneas; y el teléfono desarrollado en 1876 por Antonio Meucci, que consistía en un dispositivo de telecomunicación diseñado para transmitir conversaciones por medio de señales eléctricas (Uriarte, 2020).

Durante la segunda revolución industrial se llevaron a cabo importantes investigaciones sociales, políticas, económicas, científicas y tecnológicas a fin de mejorar la vida de las personas. Entre ellas podemos encontrar:

- Fuentes de energía. En el campo de la electricidad se destacaron los inventores Nikola Tesla y Thomas Alva Edison, este último que creó la bombilla eléctrica. Asimismo, con el descubrimiento del petróleo y sus derivados se 
creó el motor de combustión interna, entre los investigadores se destacó el ingeniero alemán Rudolf Diésel.

- Avances e invenciones tecnológicas. Los avances tecnológicos en esa época incluyeron nuevos sistemas de transporte, como aviones fabricados por los hermanos Wright (utilizados en la Primera Guerra Mundial), automóviles, ferrocarriles eléctricos y barcos propulsados por calderas. En cuanto a las comunicaciones, Samuel Morse creó el telégrafo, Alexander Graham Bell creó el teléfono, y los hermanos Lumiere desarrollaron la cinematografía (no se transmite la imagen del sonido), y la radio se destaca.

- Avances e invenciones científicas. También hay avances importantes en la ciencia, incluida la teoría de la evolución de Charles Darwin, el proceso de pasteurización y conservación de alimentos de Luis Pasteury el descubrimiento de la tuberculosis por Robert Cosme. Del mismo modo, los científicos han descubierto cómo utilizar ciertos metales, como el aluminio, el zinc o el cobre, así como diversos materiales químicos que se utilizan para fabricar fertilizantes (incluidos los explosivos) en las grandes industrias (SIGNIFICADOS,2019).

Con respecto a los sistemas de manufactura o producción, durante el desarrollo de la segunda revolución industrial se requirieron nuevos sistemas organizativos debido a la complejidad del entramado empresarial y de los procesos de producción que se manifestaron durante la segunda fase de la industrialización (Lozano, 2004).

Por ejemplo, las nuevas industrias nacieron como consecuencia de los grandes inventos, como las máquinas automáticas capaces de fabricar piezas para otras maquinarias (proceso denominado automatización de las máquinas), que permitió mejorar la competitividad en el mercado tras acelerar los tiempos de producción y reducir los costos (porque requería menos mano de obra). Además, con la bú squeda de mejorar los sistemas de producción, aumentar la productividad al nivel máximo, minimizar los tiempos y costos de producción, se implementó un nuevo sistema de producción que se basó en la idea de Frederick Winslow Taylor, donde propuso que cada obrero debía realizar la parte de una pieza dentro de una cadena de montaje y en un tiempo determinado. Todo esto se lograría eliminando pasos y movimientos innecesarios, aislamiento conveniente del operario, factibilidad para el alcance de elementos y separación del trabajo intelectual y manual, todo esto llevado conjuntamente con un riguroso cronometraje de cada tarea del proceso a fin de evitar el descuido o pérdida de tiempo del operario (Lozano, 2004).

La producción en cadena representó un avance significativo en la era industrial del siglo XX. Aplicado con éxito en las fábricas de au tomóviles de Henry Ford, donde se implantó el primer modelo de una línea de montaje de producción en masa para ensamblar coches a gran escala. Esto provocó una revolución en la industria, redu ciendo el costo de fabricación de au tomóviles A partir de ahí, la producción en masa se hizo popular en todos los campos industriales (INFAIMON, 2018).

Como se sabe, la segunda revolución industrial se caracterizó por un enorme crecimiento en muchas áreas de la industria: la minería, el transporte y la construcción, por nombrar sólo algunas. Este crecimiento creó una demanda de más materias primas y, en muchos casos, de nuevos materiales con mejores propiedades que aún no existían. Lo que originó el desarrollo de nuevos materiales, donde surgieron nuevas aplicaciones para hacer uso de ellos, creando nuevas demandas y así cíclicamente.

\section{Tercera revolución}

Mientras que la primera y segunda revolución industrial tuvo un impacto en la sociedad y en la manera de producir y fabricar nuevos productos, la tercera revolución se enfocó en la economía de los diversos países donde se llevó a cabo esta transformación (González, 2021).

Según Rifkin en su obra "La Tercera Revolución Industrial" publicada en 2011 existieron tres pilares que conformaron esta transformación, el primero fue el uso y el aprovechamiento de energías renovables, ya que descubrieron y razonaron el impacto que tiene el uso de energías no renovables en el ambiente, en segundo lugar, se encuentra la construcción de edificios que sean capaces de producir su propia energía y, por último, fue el uso del hidrógeno enfocado como un elemento importante de almacenamiento de energía (Lastra, 2017). Esta tercer etapa de industrialización tiene en su desarrollo la automatización y el uso de las tecnologías de la información, por lo que representó un gran cambio en las industrias, gracias a la automatización de los procesos productivos, las industrias tuvieron una actualización en cuanto a su maquinaria y de igual manera de su personal, debido a que sus trabajadores tenían que tener conocimiento sobre las TIC para la ejecución de diversos procesos, ya que toda la maquinaria contaba con algún código o programa para su ejecución, dando la tarea a las empresas de capacitar a los operadores de maquinaria (Lastra, 2017). 

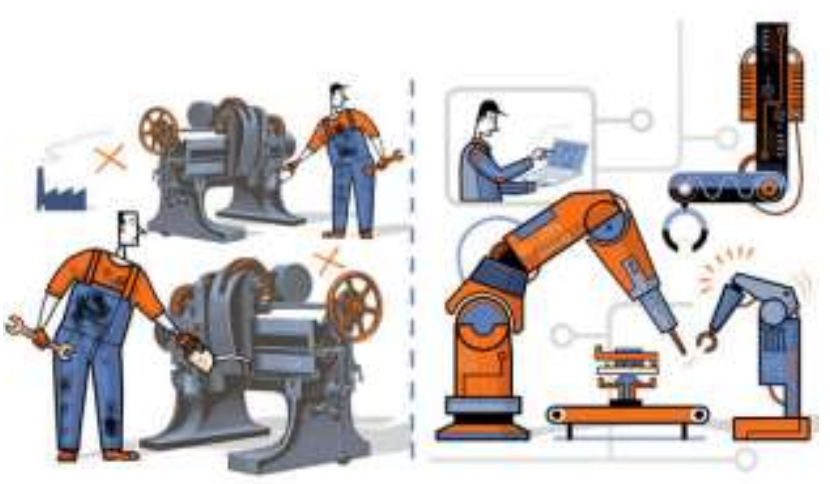

Figura 4. Transición a la tercera revolución industrial Fuente: Pampillón (2012)

Cabe señalar que la tercera revolución industrial se caracteriza principalmente por las innovaciones tecnológicas, ya que tuvo un gran impacto principalmente en la industria electrónica. La demanda de los productos durante el desarrollo de Industria 3.0 presentó un aumento a tres dimensiones, las cuales fueron: el volumen, la variedad y el tiempo de entrega. Se puede llamar a este entorno de demanda como mercado volátil, que trajo consigo fallos en la línea de flujo, obligando a la industria a tener un desarrollo tecnológico factible para poder seguir cumpliendo con las demandas que surgían día a día (Schwab K, 2017).

Gracias a las fallas que se produjeron en algunos tipos de producción en líneas de ensamblaje, es cómo fue posible la idea de crear más máquinas que pudieran ser programables, con el fin de que se pudieran cubrir las diversas necesidades de los clientes. Durante este periodo surgieron diversos desarrollos tecnológicos, iniciando con pequeñas mejoras en computadoras y finalizando con la creación del internet en los años noventa (Hasan Tinmaz, 2021).

Debido a los grandes cambios en el hardware y al incremento de la constante demanda de la automatización en diversas industrias, se logró impulsar el desarrollo de nuevos productos de software para la fabricación de productos (Yin, Stecke y Li, 2017).

Durante este periodo surgió un nuevo tipo de energía, que fue la nuclear, de modo que el ser humano tuvo la oportunidad de ser testigo del poder que conlleva este tipo de energía, ejemplo de ello fueron las bombas atómicas, demostrando así cuán dañino puede ser este tipo de energía tanto para el medio ambiente y para los animales (Yin, Stecke y Li, 2017).

Esta etapa también se caracteriza por el cuidado del ambiente, debido a que surgió la necesidad de buscar nuevas energías, que fueran amigables con el ambiente y que cubrieran las necesidades que exigía la industria en ese entonces. Algunos ejemplos de los tipos de energía son:
- La generación eólica es bastante sencillay se ha utilizado como energía fuentes durante cientos de años. Se coloca una gran hélice en el camino del viento, la fuerza del viento lo hace girar, un acoplamiento de engranajes interactúa con un se genera y captura bina y electricidad.

- Los sistemas de generación solar capturan la luz solar, incluida la radiación ultravioleta a través de células solares.

- La biomasa es un proceso químico notable que convierte los azúcares vegetales (como maíz) en gases (etanol o metano), que luego se queman o utilizan para generar electricidad.

- La energía geotérmica es la energía extraída del calor almacenado en la tierra, que se origina en la formación del planeta, la desintegración radiactiva de minerales y energía solar absorbida en la superficie.

Gracias a la búsqueda de estas nuevas formas de generar energía surgió la necesidad de crear nuevos softwares que pudieran cubrir con las necesidades de la generación de los distintos tipos de energía. (Clark II y Cooke, 2014).

La producción durante este periodo fue automatizada, dando como necesidad la capacitación de los trabajadores, como fue anteriormente mencionado, teniendo un gran impacto tanto en la economía de las industrias como en la de los trabajadores.

De igual manera, los sistemas de manufactura fueron desarrollándose de la mano de la tecnología, ya que las necesidades tecnológicas de la industria así lo requirieron.

\section{Cuarta revolución}

La cuarta revolución industrial, también conocida como Industria 4.0, describe un conjunto de transformaciones que están en marcha en nuestra economía, sociedad y forma de vivir (SALESFORCE LATINOAMÉRICA, 2021). Esta nueva era de la ciencia y la tecnología se verá desarrollarse ahora y en futuros años, pero se prevé que existan muchas uniones de las diferentes tecnologías y descubrimientos existentes, para poder innovar día con día los procesos para la elaboración de productos y hacer de ellos procesos más óptimos.

Como la cuarta revolución industrial aún no ha llegado a su fin no se pueden definir con exactitud sus características, sin embargo, se espera implementar el uso de las tecnologías como Internet de las cosas, robototronics, la producción totalmente automatizada eliminando al ser humano de los procesos de producción, la inteligencia artificial, el uso simultáneo de posibilidades de globalización y minimización de las consecuencias 
sociales negativas y la interacción global de empresas (SALESFORCE LATINOAMÉRICA, 2021).

Gracias a esto, las industrias podrían ubicarse en regiones deshabitadas, logran do un impacto menory casi nulo en la población; además, de mejorar sus líneas de producción, siendo capaces de cambiarla en poco tiempo si se requiere para producir nuevos productos. También se daría un cambio en la esencia de las patentes industriales, pues ya no se tendrá el miedo de que alguien robe la creación de algún producto o método de elaboración; ya que dicha información será visible a todo público, pero solo pocos podrán ser los que los utilicen o implementen (Popkova, Ragulina y Bogoviz, 2019).

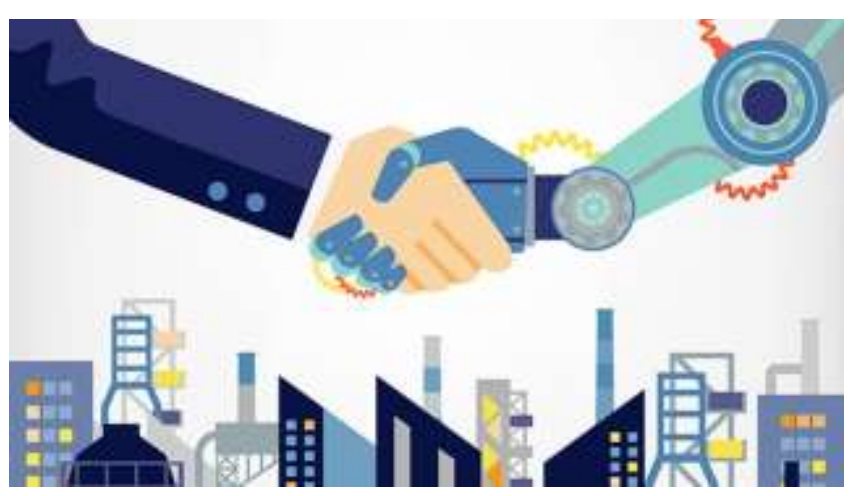

Figura 5. Revolución 4.0

Fuente: DestinoNegocio (2021)

Con respecto a la tecnología más destacada hasta el momento de esta revolución es: el Internet de las cosas (IoT), que permite el uso generalizado de la computación integrada y la estandarización de la comunicación de red digital, y la inclusión de sistemas ciber-físicos (SCF); brindando un entorno de fabricación colaborativa totalmente conectada con la recopilación y el procesamiento de los datos automatizados (Popkova, Ragulina y Bogoviz, 2019).

Sin embargo, también se encuentran hasta el momento otras tecnologías derivadas de la Inteligencia Artificial como: los Cobots, diseñados para poder interactuar físicamente con los humanos en entornos colaborativos y que buscan optimizar la producción; además de sustituir a los empleados de aquellas tareas difíciles o peligrosas. La realidad aumentada y virtual: son tecnologías que combinan el mundo real y digital, y permiten mejorar la experiencia virtual de las personas. Big data: que permite la gestión e interpretación de los datos, logrando facilitar la creación de estrategias comerciales y la toma de decisiones. La impresión 3D y 4D: que da una mejor perspectiva al momento de crear prototipos y realizarlos de forma más fácil y práctica (IBERDROLA, 2021).
Por otra parte, los tipos de sistemas de producción o manufactura (SM) que se han desarrollado en la Industria 4.0, son: los sistemas de fabricación flexible (SMF), los sistemas de fabricación celular (SMC), los sistemas de fabricación ágil (SMA) y los sistemas de fabricación celular virtual (SMCV), que son descritos con enfoque en aspectos de concepto, diseño y modelado. Donde el Internet de las cosas (IoT) se está integrando en los sistemas de manufactura con el objetivo de integrar las cadenas de suministro y los sistemas de fabricación por medio de la relación de dispositivos físicos (integrados con electrónica, software, sensores, actuadores y comunicación de red). A esta integración se conoce como Sistemas Ciber-Físicos (SCF) donde su conectividad es inalámbrica para comunicarse y coordinarse por medio del loT para fabricar productos personalizados (Abdi et al., 2018). Abdi et al. (2018) menciona que los principios del diseño de la Industria 4.0 son:

- Interoperabilidad: Donde los SCF, las personas y empresas, están conectados por medio de redes de comunicaciones.

- Descentralización: Donde los SCF son capaces de tomar decisiones autónomas.

- Modularidad: Donde los SCF están diseñados para ser el modular con características de conecta y reproduce.

- Tiempo real: Donde se da el monitoreo de las condiciones de la fábrica y los datos de recursos.

- Virtualización: Donde los datos de la fábrica y recursos, se actualizan en un modelo virtual para dar seguimiento y simulación al modelo.

- Orientación al servicio: Donde los SCF, las personas y empresas ofrecen sus servicios a través de Internet de servicios (loS) de forma similar a la fabricación en la nube.

De tal forma que, el loT se utiliza para coordinarlas tareas entre los recursos para obtener decisiones descentralizadas, autónomas y basadas en el conocimiento (automatización) (Abdi et al., 2018).

Junto con la digitalización y la revolución de las Tecnologías de la Información (TI), las fábricas inteligentes del futuro, aún no se han realizado satisfactoriamente; por ello, la Industria 4.0 se puede ver como una fusión del mundo real y virtual por medio de los sistemas de producción ciber-físicos (SPCF) o redes en línea en las que las $\mathrm{TI}$ y las máquinas están vinculadas. Es por eso que, las TI vinculadas a la Industria 4.0 y las cadenas de suministro son muy importantes, pues se puede manejar de mejor manera el intercambio electrónico de datos (IED), la identificación de radiofrecuencia (IRF), el código de barras, el comercio electrónico, el sistema de apoyo a la toma de decisiones 
y la planeación de los recursos empresariales (ERP) (Abdi et al., 2018).

\section{Tendencias de los sistemas de manufactura y producción en la Industria $\mathbf{4 . 0}$}

La Industria 4.0, también llamada industria inteligente, se considera la cuarta revolución industrial y busca principalmente transformar a la empresa en una organización inteligente para conseguir los mejores resultados, todo esto implementado desde su planificación, ejecución y control de la producción. Así mismo esta nueva etapa en la industria apuesta por una mayor automatización, conectividad y globalización (CIC, 2017).

La cuarta revolución sin duda, se verá caracterizada por la adopción de las nuevas tecnologías para la progresiva automatización de los procesos productivos. Se trata de tecnologías innovadoras cuya aplicación a la industria se desarrollará día a día. Se habla de fabricación robótica colaborativa, con herramientas de planeación de la producción, visión artificial, realidad virtual, simulación de procesos y programas modernos de diseño, impresiones 3D, la nano y la biotecnología, la inteligencia operacional, loT, y las denominadas Tecnología facilitadoras esenciales (KET, por sus siglas en inglés) (CIC, 2017).

En un futuro no muy lejano se vivirá una realidad donde las fábricas inteligentes integrarán lo físico con lo virtual y digital, y dónde los fabricantes y máquinas compartirán información en un formato comprensible para todos los usuarios promovien do la colaboración reforzada y el uso compartido de datos en las cadenas de suministro, además de que los procesos podrán ser optimizados automáticamente, ser auto-configurables y usar inteligencia artificial para completar tareas difíciles basadas en flujos de trabajo complejos. Así mismo, la automatización de los procesos de mantenimiento y la inteligencia artificial, serán imprescindibles para esta gran transformación (ClC, 2017).

La combinación de automatización y fabricación asistida por computadora tendrá gran impacto en la manufactura y el enfoque de la producción en las empresas inteligentes, ya que les permitirá desarrollar sistemas de fabricación avanzados que serán utilizados en la administración de la producción y las operaciones, como el MRP, el ERP y el sistema de ejecución de fabricación, todos enfatizados en la planeación, programación, ejecución y control de la producción en términos de costo, eficiencia y calidad (CIC, 2017).

Todo esto implicará la necesidad de disponer de sistemas que operen y gestionen la información de banda ancha, así como infraestructura para las tecnologías de la información. El entorno será aún más inteligente que ahora y planteará muchas oportunidades, pero también retos a lo largo de esta Industria 4.0. Por ejemplo, la creación de nuevos productos inteligentes que presenten valores añadidos respecto a la competencia e impacten positivamente en el bienestar personal. El tema de ciberseguridad es otro ejemplo, será una de las cuestiones clave para el mundo industrial interconectado. Por supuesto, la revolución industrial también modifica el perfil de recursos humanos que se va a necesitar en las nuevas fábricas: se pedirán las habilidades y los conocimientos diferentes de lo que se piden en la actualidad, además de que la dinámica del trabajo también será diferente. Este concepto de Industria 4.0 representará un salto muy importante para la mayoría de las organizaciones (CIC, 2017).

La cuestión que se observará es que las industrias se enfrentarán un gran reto, deberán buscar no quedarse atrás y prepararse para los cambios que traerá el desarrollo de la cuarta revolución industrial; para las empresas no se tratará de algo tecnológico, si no en el saber gestionar el lanzamiento de programas que pretendan llevar a la industria hacia el futuro, saber aprovechar al máximo las nuevas oportunidades que surgirán y apoyar todo tipo de formación en cuanto a la adaptación a esta nueva realidad que exige más liderazgo y cambios organizativos para llevar a cabo una transformación empresarial (CIC, 2017).

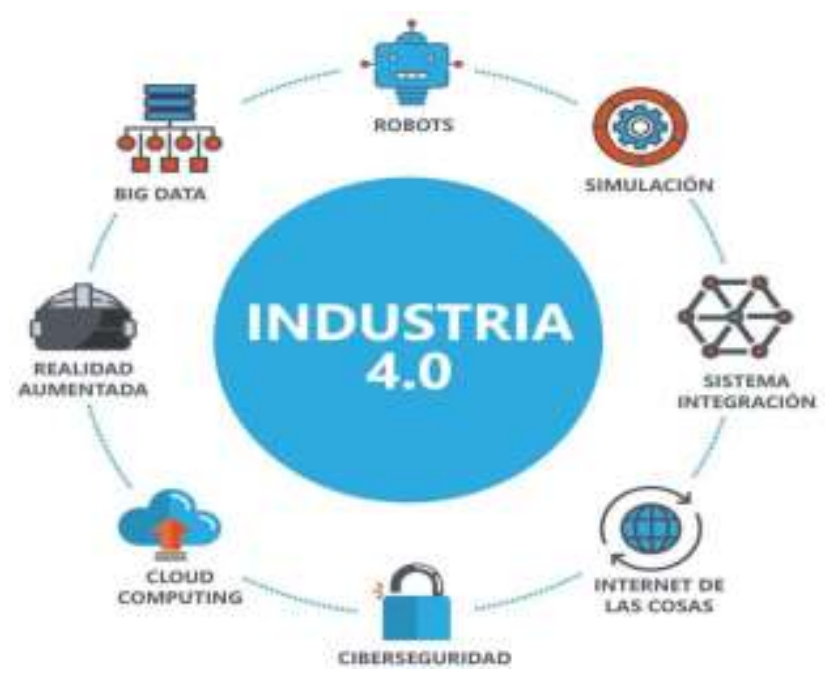

Figura 6. Industria 4.0 y manufactura flexible

Fuente: COMECA (2018)

\section{Conclusión}

A partir del crecimiento exponencial de la población en todo el mundo, la demanda por alimentos, vestimenta, medicinas, productos o servicios también se han amplificado de la misma forma. Por lo tanto, las personas $y$ las empresas han cambiado sus procesos y procedimientos de producción para superar esas inmensas demandas. A finales del siglo XVIII, la humanidad se enfrentó al primer desarrollo tecnológico conocido como primera revolución industrial, pasando de un sistema de producción artesanal (independiente o coordinada por un comerciante) a una producción en masa (período mecánico). Para el siglo XIX la electricidad supera al vapor de muchas maneras, es más económico y se puede transmitir rápidamente a larga distancia con pocas pérdidas. Su amplia aplicación llevó a la sociedad 
humana de "la era del vapor" a "la era de la electricidad", conocida como segunda revolución industrial.

En la segunda mitad del siglo XIX, se inventaron muchos productos y tecnologías eléctricos, como luces, tranvías eléctricos, taladros eléctricos y soldadura eléctrica, etc. De tal forma que, la segunda revolución expandió la industrialización a las industrias pesadas, como el hierro y el acero, y la construcción de maquinaria, entre otras. Además, surgieron y se desarrollaron algunas industrias nuevas, como la del petróleo, química, eléctrica y automotriz (la invención del automóvil fue un evento importante esta revolución Industrial). En el contexto de los sistemas de producción, la productividad mejoro y la precisión del mecanizado se convirtió en el tema central de la fabricación, propiciando el desarrollo de la producción en masa, y la estandarización y serialización se mejoraron gradualmente. Además, se desarrolló la primera línea de ensamblaje del mundo (En 1913 por Ford Motor Company). Por otra parte, en este periodo se sientan las bases de la administración científica, a partir de los trabajos de Frederick Winslow Taylor, considerado como el fundador de la administración científica de la que surgió una nueva disciplina, la ingeniería industrial.

Una vez que la humanidad tiene el dominio y el poder de la electricidad, la gente y las empresas producen más dispositivos con enormes capacidades, como computadoras, robots e Internet. En ese período conocido como la tercera revolución industrial (también llamado período de las computadoras), se han diseñado, producido, implementado e integrado muchas tecnologías de la información y la comunicación no solo en las diferentes industrias sino también en la vida cotidiana de muchas personas.

En los sistemas de manufactura se propicia el uso de la computadora para los procesos de producción y se generan sistemas de fabricación más automatizados. Es decir, las instalaciones de fabricación se convierten en más flexibles con herramientas y accesorios intercambiables, en donde se caracteriza por la fabricación flexible, la producción esbelta (lean manufacturing), por el uso de celdas de producción vinculadas, producción justo a tiempo, fabricación integrada por computadora (CAD-CAM), fabricación ágil y fabricación aditiva. Estos cambios tecnológicos, técnicos y administrativos permitieron en los sistemas de producción la reducción de los tiempos de preparación, se minimiza el inventario (se minimizan los costos de manipulación de materiales), se simplifica la programación de la producción y se mejora la distribución de los productos y servicios y se mejora la calidad.

Finalmente, la cuarta revolución industrial o Industria 4.0 es el paradigma más nuevo del desarrollo humando. En donde se caracteriza por búsqueda de la sostenibilidady la ventaja competitiva de las empresas conectadas a cadenas de suministro globales. Además, este nuevo paradigma se centra en la fabricación inteligente, en donde destacan los sistemas ciberfísicos (dispositivos mecatrónicos con conectividad inalámbrica que se comunican y coordinan entre sí a través del Internet de la Cosas) para fabricarproductos personalizados. En donde el Internet de las cosas se utiliza para la integración de cadenas de suministro y sistemas de fabricación a través de la interconexión de dispositivos físicos, integrados con electrónica, software, sensores, actuadores y comunicación de red.

\section{Referencias.}

Alcácer, V. \& Cruz-Machado, V. (2019). Scanning the Industry 4.0: A Literature Review on Technologies for Manufacturing Systems. Engineering Science and Technology, an International Journal, 22(3), 899-919.

Abdi, M. R., Labib, A. W., Edalat, F. D. \& Abdi, A. (2018). Evolution of MS Paradigms Through Industrial Revolutions. In: Integrated Reconfigurable Manufacturing Systems and Smart Value Chain. Springer, Cham.

Avansis. (2021). Industria 4.0 ¿es realmente revolución o evolución?. Recuperado el 19 de marzo de 2021, de https://www.avansis.es/industria-4-0/industria-4-0-revolucionevolucion/

BARDAHL. (2020). ¿Cómo Cambió la Revolución Industrial a la Manufactura?. Recuperado el 19 de marzo de 2021, de https://www.bardahlindustria.com/como-cambio-la-revolucionindustrial-a-la-

manufactura/\#: : text=Antes\%20de\%20la\%20Revoluci\%C3\%B3n\%20 Industrial,demanda\%20controla da $\% 20$ de $\% 20$ sus $\% 20$ productos.

Bembibre, C. (2014). Revolución Industrial. Recuperado el 19 de marzo de 2021 https://www.importancia.org/?s=Revoluci\%C3\%B3n\%20Industrial.

Chávez Palacios, J. (2014). Desarrollo tecnológico en la primera revolución industrial. Dialnet.unirioja.es. Recuperado, 23 April 2021, de https://dialnet.unirioja.es/descarga/articulo/1158936.pdf.

Coluccia, D. (2012) The First Industrial Revolution (c1760-c1870). In: Zanda G. (eds) Corporate Management in a Knowledge-Based Economy. Palgrave Macmillan, London.

CIC-Consulting Informático. (2017). Industria 4.0, la cuarta revolución industrial y la inteligencia operacional. Recuperado el 19 de marzo de 2020, de https://www.cic.es/industria-40-revolucion-industrial/.

Clark II, W. W. y Cooke, G. (2014). The Green Industrial Revolution. Butterworth-Heinemann.

COMECA. (2018). QUÉ ES LA INDUSTRIA 4.0. Recuperado el 19 de marzo de 2021, de https://comeca.com.mx/que-es-la-industria-4-0/.

Dos Santos, E. F., \& Dos Santos Nunes, L. (2017). Methodology of risk analysis to health and occupational safety integrated for the principles of lean manufacturing. Advances in Intelligent Systems and Computing, $487,349-353$.

Doll, W., \& Vonderembse, M. (1991). The evolution of manufacturing systems: Towards the post-industrial enterprise. Omega, 19(5), 401411.

DestinoNegocio. (2021). ¿Qué es la Cuarta Revolución Industrial?. Recuperado el 19 de marzo de 2021, de https://destinonegocio.com/mx/gestion-mx/que-es-la-cuartarevolucion-industrial/.

Easterlin, R. A. (2019). Three Revolutions of the Modern Era. Comparative Economic Studies, 61(4), 521-530. 
Ebrahimi, M., Baboli, A., \& Rother,E. (2018). A Roadmap for evolution of existing production system toward the factory of the future: Aca se study in automotive industry. 2018 IEEE International Conference on Technology Management, Operations and Decisions, ICTMOD 2018, 274-281.

González, I. (2021). Revolución Industrial: Aprendiendo a Evolucionar. Recuperado el 19 de marzo de 2021, de https://predictiva21.com/revolucion-industrialmexico/\#: :text=La \%20revoluci\%C3\%B3n\%20industrial\%20se $\% 20 \mathrm{c}$ onoce, hasta $\% 20$ inicios $\% 20$ del $\% 20$ siglo $\% 20$ XIX.\&text=Las $\% 20$ cond cione $\% 20 \mathrm{de} \% 20$ trabajo\%20tambi\%C3\%A9n,del\%20producto\%20de $\% 20$ esta\%20revoluci\%C3\%B3n.

Guo, D., Li, M., Zhong, R., \& Huang, G. Q. (2020). Graduation Intelligent Manufacturing System (GiMS): an Industry 4.0 paradigm for production and operations management. Industrial Management and Data Systems, 121(1), 86-98

INFAIMON. (2018). Producción en cadena: evolución y ventajas. Recuperado el 19 de marzo de 2021, de https://blog.infaimon.com/produccion-en-cadena-evolucion-ventajas/.

IBERDROLA. (2021). Industria 4.0: ¿qué tecnologías marcarán la Cuarta Revolución Industrial?. Recuperado el 19 de marzo de 2021, de https://www.iberdrola.com/innovacion/cuarta-revolucion-industrial

KAPELUSZ (2020). La segunda revolución industrial. Recuperado el 19 de marzo de 2021, de https://www.editorialkapelusz.com/wpcontent/uploads/2018/02/CAP-MODELO-AVANZA-SOCIALESFEDERAL-6.pdf

Khan, A., \& Turowski, K. (2016). A perspective on industry 4.0: From challenges to opportunities in production systems. IoTBD 2016 Proceedings of the International Conference on Internet of Things and Big Data, IoTBD, 441-448.

Kim, J. Y., Kim, H., \& Lee, J. S. (2016). An analysis of manufacturingservice industry co-evolution: A spiral growth model. Journal of Service Science Research, 8(1), 57-72.

Kumar, A., \& Kumar, S. (2018). Review of Trends and development in flexible and reconfigurable manufacturing systems. International Research Journal of Engineering and Technology (IRJET), 5(4), 4-9.

Kumar, G., KumarGoyal, K., \& Batra, N. K. (2019). Evolution, principles and recent trends in reconfigurable manufacturing system. Journal of Physics: Conference Series, 1240(1).

Lastra, J. (2017). La Tercera Revolución Industrial. Boletín Mexicano de Derecho Comparado, (150), 1457-1462.

Lin, Y., Luo, J., Ieromonachou, P., \& Huang, L. (2018). Manufacturing System Evolution. 2018 15th International Conference on Service Systems and Service Management, ICSSSM 2018, 1-6.

Lozano J. J. C. (2004). La revolución industrial. Recuperado el 19 de marzo de 2021, de http://www.cla seshistoria.com/revolucionindustrial/2formasorganizaci on.htm\#arriba

Mehrabi, M. G., Ulsoy, A. G., Koren, Y. \& Heytler, P. (2002). Trends and perspectives in flexible and reconfigurable manufacturing systems. Journal of Intelligent Manufacturing , 13, 135-146.

Mokyr, J. (1999). The Second Industrial Revolution, 1870-1914 Joel. In Valerio Castronovo, Ed., Storia Dell'economia Mondiale. Rome: Laterza Publishing, 847, 219-245.

Monllor, J. (2012). Segunda revolución industrial. Recuperado el 19 de marzo de 2021, de https://sites.google.com/site/geografia2bat/historiacontemporanea/5-segunda-revolucion-industrial

Nakayama, R. S., de Mesquita Spínola, M., \& Silva, J. R. (2020). Towards I4.0: A comprehensive analysis of evolution from I3.0. Computers and Industrial Engineering, 144(March), 106453.

Pampillón, R. (2012). La tercera revolución industrial. Recuperado el24 de abril de 2021, de https://economy.blogs.ie.edu/archives/2012/08/latercera-revolucion-industrial/.
Pozdnyakova, U. A., Golikov, V. V., Peters, I. A., \& Morozova, I. A. (2019). Genesis of the revolutionary transition to industry 4.0 in the $21 \mathrm{st}$ century and overview of previous industrial revolutions. Studies in Systems, Decision and Control, 169, 11-19.

Popkova, E. G., Ragulina, Y. V., \& Bogoviz, A. V. (2019). Fundamental differences of transition to industry 4.0 from previous industrial revolutions. Studies in Systems, Decision and Control, 169, 21-29.

QAlovers. (2017). La teoría del Taylorismo. Recuperado el 19 de marzo de 2021, de https://www.qa lovers.com/2017/02/taylorismo.html

Riedel, D. (2016). ¿Blockchain impulsará la cuarta revolución industrial?. Recuperado el 18 de marzo de 2021, de https://readwrite.com/2016/05/09/blockhain-new-ir/.

Selva V. B. (2016). Revolución industrial. Recuperado el 19 de marzo de 2021, de https://economipedia.com/definiciones/primera-revolucionindustrial.html\#: :text=La\%20Revoluci\%C3\%B3n\%20Industrial\%20 marc\%C3\%B3\%20un,los\%20\%C3\%A1 mbitos\%20de\%20la\%20socied ad.\&text $=$ En $\% 20 \operatorname{los} \% 20$ siglos $\% 20 X X \% 20 y$, la $\% 20$ Cuarta $\% 20$ Revoluc i\%C3\%B3n\%20Industria1\%2C\%20respectivamente.

SIGNIFICADOS (2020). Segunda revolución industrial. Recuperado el 19 de marzo de 2021, de https://www.significados.com/segunda revolucion-industrial/.

Tinmaz, H. (20). History of Industrial Revolutions: From Homo Sapiens Hunters to Bitcoin Hunters. In: Rosa Righi R., Alberti A., Singh M. (eds) Blockchain Technology for Industry 4.0. Blockchain Technologies. Springer, Singapore.

SALESFORCE LATINOAMÉRICA. (2021). Qué es la Cuarta Revolución Industrial?. Recuperado el 19 de marzo de 2021, de https://www.salesforce.com/mx/blog/2018/4/Que-es-la-CuartaRevolucion-Industrial.html.

Uriarte J.M. (2020). Segunda revolución Industrial. Recuperado el 19 de marzo de 2021, de https://www.caracteristicas.co/segunda-revolucionindustrial/.

Yin, Y., Stecke, K. E., y Li, D. (2017). The evolution of production systems from Industry 2.0 through Industry 4.0. International Journal of Production Research, 56(1-2), 848-861

Zhang, C., y Yang, J. (2020). A History of Mechanical Engineering. In A History of MechanicalEngineering. Springer, Singapore. 